\title{
Dissociated lateralization of transient and sustained blood oxygen level-dependent signal components in human primary auditory cortex
}

\author{
Christoph Lehmann, ${ }^{\mathrm{a}, \mathrm{b}, *, 1}$ Marcus Herdener, ${ }^{\mathrm{a}, \mathrm{c}, 1}$ Peter Schneider, ${ }^{\mathrm{g}}$ Andrea Federspiel, ${ }^{\mathrm{a}}$ \\ Dominik R. Bach, ${ }^{a}$ Fabrizio Esposito, ${ }^{\mathrm{d}}$ Francesco di Salle, ${ }^{\mathrm{e}}$ Klaus Scheffler, ${ }^{\mathrm{f}}$ \\ Robert Kretz, ${ }^{\mathrm{b}}$ Thomas Dierks, ${ }^{\mathrm{a}}$ and Erich Seifritz ${ }^{\mathrm{a}}$
}

${ }^{\mathrm{a}}$ University Hospital of Clinical Psychiatry, University of Bern, 3000 Bern, Switzerland

${ }^{\mathrm{b}}$ Neuroanatomy, Department of Medicine, University of Fribourg, 1700 Fribourg, Switzerland

${ }^{\mathrm{c}}$ Forensic-psychiatric Service, University of Bern, 3012 Bern, Switzerland

'Department of Neurological Sciences, University of Naples "Federico II", 80127 Naples, Italy

e Department of Neuroscience, University of Pisa, 56126 Pisa, Italy

${ }^{\mathrm{f}}$ MR-Physics, Department of Medical Radiology, University of Basel, 4031 Basel, Switzerland

${ }^{\mathrm{g}}$ Department of Neurology, University Hospital Heidelberg, INF 400, D-69120 Heidelberg, Germany

\begin{abstract}
Among other auditory operations, the analysis of different sound levels received at both ears is fundamental for the localization of a sound source. These so-called interaural level differences, in animals, are coded by excitatory-inhibitory neurons yielding asymmetric hemispheric activity patterns with acoustic stimuli having maximal interaural level differences. In human auditory cortex, the temporal blood oxygen level-dependent (BOLD) response to auditory inputs, as measured by functional magnetic resonance imaging (fMRI), consists of at least two independent components: an initial transient and a subsequent sustained signal, which, on a different time scale, are consistent with electrophysiological human and animal response patterns. However, their specific functional role remains unclear. Animal studies suggest these temporal components being based on different neural networks and having specific roles in representing the external acoustic environment. Here we hypothesized that the transient and sustained response constituents are differentially involved in coding interaural level differences and therefore play different roles in spatial information processing. Healthy subjects underwent monaural and binaural acoustic stimulation and BOLD responses were measured using high signal-to-noise-ratio fMRI. In the anatomically segmented Heschl's gyrus the transient response was bilaterally balanced, independent of the side of stimulation, while in opposite the sustained response was contralateralized. This dissociation suggests a differential role at these two independent temporal response components, with an
\end{abstract}

\footnotetext{
* Corresponding author. University Hospital of Clinical Psychiatry, Department of Psychiatric Neurophysiology, Bolligenstrasse 111, CH3000 Bern 60. Fax: +41 319309961.

E-mail address: lehmann@puk.unibe.ch (C. Lehmann).

1 These authors contributed equally to this work.
}

initial bilateral transient signal subserving rapid sound detection and a subsequent lateralized sustained signal subserving detailed sound characterization.

Keywords: Auditory system; Sound localization; Lateralization; Imaging; fMRI; Statistical modeling

The capability of the auditory system to detect changes in the three-dimensional space of the environment is essential to detect potential danger. The spatial representation of the acoustic environment, i.e. the localization of acoustic sources, needs to be reconstructed from time-series information received by the two ears (Zatorre, 2003), since in contrast to the visual system there is no point-to-point topographic organization of the auditory cortex. Among other physical information, the brain uses interaural time differences (ITD) and interaural level differences (ILD) to help localize sounds (Moore, 1994), binaural cues known to be separately processed in subcortical (Caird and Klinke, 1983; Joris and Yin, 1995) and cortical (Tardif et al., 2006) regions.

In the cortex of Heschl's gyrus two types of neurons exist, which are arranged in alternating zones. In half of these strips, known as summation columns, so-called excitatory-excitatory (EE) cells are excited by stimulation of either ear, though the contralateral input is usually stronger than the ipsilateral input. The other bands, known as suppression columns, comprise so-called excitatory-inhibitory (EI) neurons, which are also excited by contralateral input but inhibited by ipsilateral input. These EI neurons are known to represent the ILD in expressing the 
difference in the coded intensity between both ears in their discharge pattern (Pollak et al., 2002). Acoustic stimulation with maximal ILD, i.e. monaural stimulation, has been shown to exhibit asymmetric hemispheric activation patterns in animal primary auditory cortex because of crossing fibers in the ascending auditory pathways and because of the inhibiting characteristic of the EI neurons in the suppression columns (for a review see Clarey et al., 1992).

In humans, many lateralization studies report contralateral dominance (Jancke et al., 2002; Krumbholz et al., 2005; Pantev et al., 1998; Scheffler et al., 1998; Woldorff et al., 1999), however a functional magnetic resonance imaging (fMRI) study constraining the signal analysis to the anatomically defined primary auditory cortex found greater activation in left compared to right primary auditory cortex, independent of the side of stimulation (Devlin et al., 2003). There are, however, only a few fMRI studies, that have segmented the blood oxygenation level-dependent (BOLD) signal in the time domain, although in auditory cortex the BOLD signal evoked by prolonged auditory stimulation can be decomposed temporally into two independent signal constituents (Harms and Melcher, 2002; Jancke et al., 1999; Seifritz et al., 2002): a phasic or transient initial response, peaking at about $5 \mathrm{~s}$ after stimulus onset and then returning to zero, and a sustained response that continues for the duration of sound stimulation. On a different time scale, studies in animals suggest the two similar signal constituents reflecting different roles in representing the external acoustic environment (Wang et al., 2005) and being originated in two different synaptic networks (Atzori et al., 2001; Zador, 2001).

Here spatial and temporal segmentation was used to test the hypothesis that sustained and transient response components measured in a-priori anatomically defined primary auditory cortices dissociate while coding interaural level differences. Six healthy subjects underwent monaural and binaural acoustic stimulation, while being measured using high signal-to-noise ratio fMRI (Seifritz et al., 2006). The BOLD signal time-courses in auditory cortex contra- and ipsilateral to the side of stimulation were deconvolved into the transient and sustained signal constituents and respective lateralization indices were computed.

\section{Materials and methods}

Subjects

Six healthy male subjects (age range 30-42 years) were recruited from an academic environment. None of the subjects had a history of major medical, neurological, or psychiatric disorders or of psychotropic medication. All subjects gave written informed consent before participation.

\section{Auditory stimulation}

Pure sine tones $(225,1000,8000 \mathrm{~Hz})$ with constant intensity of $95 \mathrm{~dB}$ sound pressure level were amplitude-modulated with a smooth square wave envelope (exponential onset and offset ramps of $10 \mathrm{~ms}$ duration) of $5 \mathrm{~Hz}$ and a duty cycle of $50 \%$. Each stimulation lasted $30 \mathrm{~s}$ and was followed by a resting period of $30 \mathrm{~s}$. The duration of the whole experiment was $20 \mathrm{~min}$ and $11 \mathrm{~s}$ (561 functional volumes). Noise reduction by the headphones (Commander XG, Resonance Technology, Northridge, CA, USA; www.mrivideo.com) of approximately $30 \mathrm{~dB}$ and the spectral composition of the scanner noise enabled a clear perception of all experimental stimuli. Sounds of the three frequencies were presented twice to the right, twice to the left and twice to both ears. Different frequencies should prevent from habituation effects over the $20 \mathrm{~min}$ lasting run. Eighteen trials were presented randomly intermixed during one single fMRI run. Subjects were instructed to concentrate on the auditory signals. They were not asked to carry out any output task.

\section{Functional imaging}

Scanning was performed on a 1.5-T MRI scanner equipped with a Sonata gradient system and a circularly polarized radio frequency head coil (Siemens, Erlangen, Germany). The subjects' heads were fixated with foam pads to minimize movement during the experiment. A $\mathrm{T}_{1}$-weighted high-resolution data set covering the whole brain was collected for each subject with a threedimensional magnetization-prepared rapid acquisition gradient echo sequence with $1 \mathrm{~mm}^{3}$ cubic voxels. To maximize the signal-to-noise ratio in auditory fMRI with preserved time resolution needed for temporal segmentation of the BOLD signal, we used continuous-sound fMRI (Seifritz et al., 2006). Twelve image slices having a thickness of $5 \mathrm{~mm}$ were measured with a volume repetition time (TR) of $2.016 \mathrm{~s}$ (field of view, $180^{2} \mathrm{~mm}^{2}$; matrix, $128^{2}$ pixels; echo time, $61 \mathrm{~ms}$; flip angle, $90^{\circ}$; bandwidth, $1280 \mathrm{~Hz} /$ pixel; slice acquisition time, $167 \mathrm{~ms}$; for technical reasons, a matrix with $64^{2}$ pixels was used for one subject, which is not of relevance, since the data was resampled into 3-mm isotropic voxels prior to statistical analysis). The functional volumes were positioned parallel to the lateral sulcus and covered the superior surface of the temporal lobe.

\section{Image processing and statistical analysis}

The first 10 volumes were discarded to allow for $T_{1}$ equilibration effects. Processing of the fMRI data was performed using BrainvoyagerQX (Brain Innovation, Maastricht, The Netherlands). The time-series were corrected for slice acquisition time through linear interpolation, realigned with their corresponding $\mathrm{T}_{1}$ volumes, warped into standard space (Talairach and Tournoux, 1988), resampled into 3-mm isotropic voxels, motioncorrected using Levenberg-Marquarts's least square fit for six spatial parameters, highpass-filtered for removal of lowfrequency fluctuations, corrected voxelwise for linear drifts, and spatially smoothed using a 8-mm full-width at half-maximum Gaussian kernel for group statistics. No spatial smoothing was performed for the individual region-of-interest (ROI) analysis in Heschl's gyrus. Prior to multi-subject statistical group analysis, the image time-series were $z$-transformed to reduce intersessionand intersubject-variance caused by the variability in the receiver sensitivity.

A general linear model (GLM) was fitted to the data (Friston et al., 1995) using condition-specific stimulus boxcar functions, convolved with a gamma-kernel (Boynton et al., 1996) to model the hemodynamic response behavior. The transient response was modelled using a boxcar of length $2 \mathrm{~s}$ thus after convolution with the gamma-kernel yielding a typical hemodynamic response peaking at about $6 \mathrm{~s}$ (Belin et al., 1999; Harms and Melcher, 2002; Lehmann et al., 2006; Seifritz et al., 2002). The sustained response was modelled by the convolution of a $30 \mathrm{~s}$ boxcar with the gamma-kernel (see Fig. 1). Six predictors entered the model: Monaural left ear stimulation transient response (LT), monaural 
BOLD signal contralateral to the side of stimulation

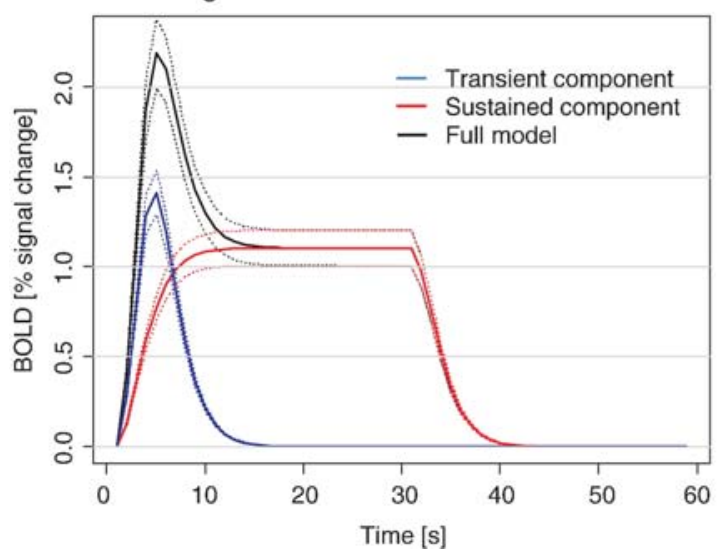

BOLD signal ipsilateral to the side of stimulation

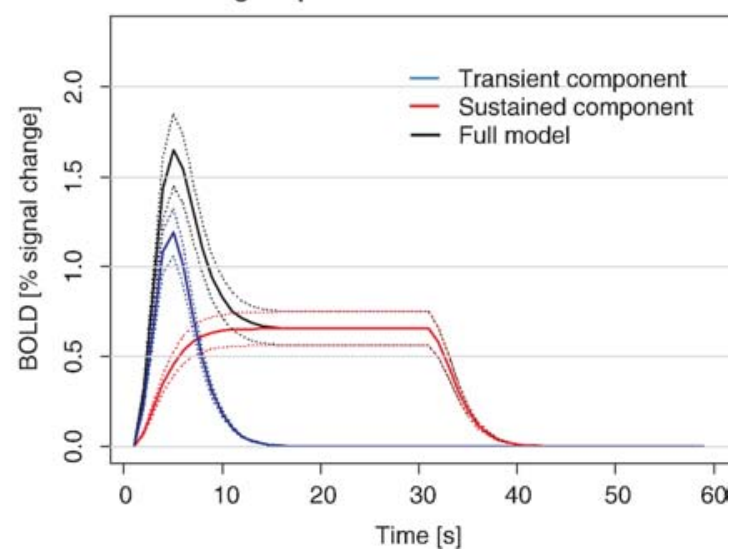

Fig. 1. General linear model estimates for transient and sustained response components in Heschl's gyrus. Left figure: the hemisphere contralateral to the side of stimulation. Right figure: hemisphere ipsilateral to the side of stimulation. Shown are the modeled components and the linear summation of these two components (full model). Dotted lines denote standard errors of the parameter estimates. The sustained response component is significantly larger in the Heschl's gyrus contralateral to the stimulated ear; however the transient response component is neither lateralized to the contra- nor to the ipsilateral side of stimulation.

left ear stimulation sustained response (LS), the same combination for monaural right ear (RT, RS) and binaural (BT, BS) stimulation.

For the investigation of lateralization, in each subject the analysis was confined to the anatomically defined primary auditory cortex, which is located in the first transverse temporal Heschl's gyrus, mainly confined to the medial two-thirds (Brodal, 1981; Schneider et al., 2002; Yousry et al., 1997). After manual grey/white matter segmentation the surface of the isolated temporal lobes was reconstructed using the surface module of the BrainvoyagerQX software. Then in each individual subject the left and right Heschl's gyrus were defined according to the definition proposed by Yousry et al. (1997). The so-called patches-of-interests, defined in the reconstructed surface, were back-transformed into the original anatomical dataset. A resampling along the grey/white matter boundary with a bandwidth of $\pm 3 \mathrm{~mm}$ yielded a mask for each individual Heschl's gyrus, used as ROI for subsequent individual quantification of lateralization.

For each subject an individual GLM (as described above) was computed in the a-priori anatomically defined gyri of heschl, corrected for serial correlation. In both gyri of heschl the time courses of all voxels with a $t$-value $>2.0$ (equals a $p$ value $<0.05$, uncorrected) were averaged and the GLM was recomputed. This yielded two sets of parameter estimates (beta values): BT, BS, LT, LS, RT, RS in right $(\mathrm{RH})$ and left $(\mathrm{LH})$ primary auditory cortex. For both, right and left ear stimulation, a laterality index (LI) was computed for each single subject based on the individual parameter estimates as follows (Devlin et al., 2003):

$\mathrm{LI}=\frac{\text { contralateral } \mathrm{BOLD} \text { signal }- \text { ipsilateral BOLD signal }}{\text { contralateral } \mathrm{BOLD} \text { signal }+ \text { ipsilateral } \mathrm{BOLD} \text { signal }} \times 100$

LI values therefore lie in the range from +100 (completely contralateral activation) to -100 (completely ipsilateral activation). The LI values from all subjects were then tested against the between-subject variability, i.e. subjects were treated as random effects and therefore our LI values entered a one sample $t$-test. To test for the interaction between the temporal component (sustained versus transient response) and the lateralization, normalized parameter estimates also entered a three-factorial analysis of variance (ANOVA; factors: side of stimulation, hemisphere, response component).

\section{Results}

Fig. 1 shows the GLM estimates for transient and sustained response components in the Heschl's gyrus in the hemisphere contralateral to the side of stimulation and the hemisphere ipsilateral to the side of stimulation. Group mean laterality indices for the transient and sustained response in individually segmented

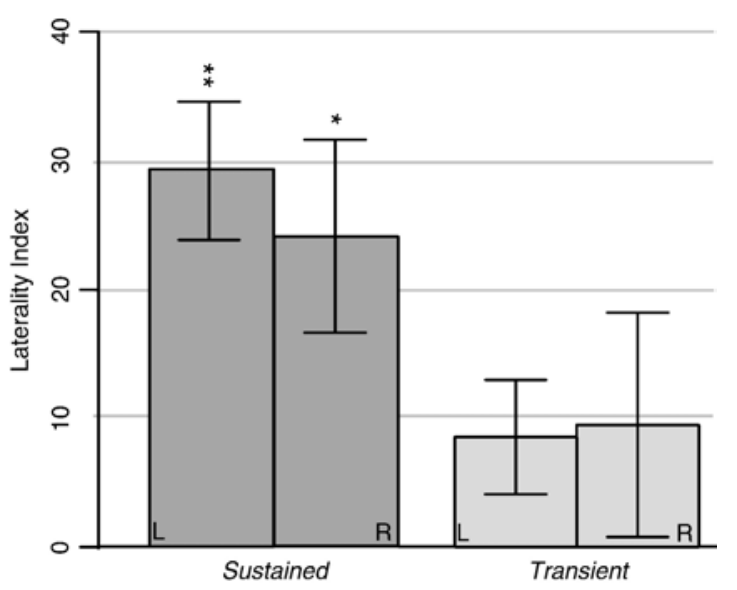

Fig. 2. Bilateral transient response versus contralateralized sustained response. Group mean laterality indices for the transient and sustained response in individually segmented gyri of heschl, obtained with monaural left (L) and right ear (R) stimulation. The bars denote standard errors of the mean. An analysis of variance (details see text) shows that the lateralization pattern of transient and sustained response component is significantly different $(p<0.01)$. Independent of the ear of stimulation, the transient response upon monaurally presented pure tones was bilateral. However, the sustained response was significantly larger in the Heschl's gyrus contralateral to the stimulated ear (see Results) $\left({ }^{*}, p<0.05 ; * *, p<0.01\right)$. 
gyri of heschl, obtained with monaural left and right ear stimulation, are shown in Fig. 2. Independent of the ear of stimulation, the transient response component is neither lateralized to the contra- nor to the ipsilateral side of stimulation (RT, $\left.t_{5}=1.094, p=0.324 ; \mathrm{LT}, t_{5}=1.927, p=0.112\right)$, however, the sustained response component is significantly larger in the Heschl's gyrus contralateral to the stimulated ear (RS, $t_{5}=3.269$, $p<0.022$; LS, $\left.t_{5}=5.465, p<0.003\right)$. For binaural stimulation no lateralization has been observed both for transient and sustained signal components (BT, $t_{5}=0.053, p<0.960 ; \mathrm{BS}, t_{5}=0.072$, $p<0.945$ ). As expected an additional three-factorial ANOVA (side of stimulation, hemisphere, response component) yielded a significant interaction between hemisphere and side of stimulation $\left(F_{1,5}=46.779, p<0.001\right)$ and a significant interaction between response component, hemisphere and side of stimulation $\left(F_{1,5}=16.993, p<0.009\right)$, confirming a significant different pattern of lateralization for transient and sustained response components.

\section{Discussion}

For the human primary auditory cortex using spatio-temporal segmentation we here have demonstrated a significant differential involvement of transient and sustained BOLD signal constituents in representing interaural level differences. Independent of the ear of stimulation, the transient response to monaurally presented pure tones was bilateral. However, the sustained response was significantly larger in the Heschl's gyrus contralateral to the stimulated ear, the dissociation being supported by a significant interaction.

This dissociation emphasizes the necessity of segmentation in the time domain, i.e. to model separately the two response components. Using four widely divergent tasks, Fox and colleagues have demonstrated that conventional block design analysis techniques that do not address separate transient and sustained signal effects, obscure important functional information about task-relevant processes (Fox et al., 2005a,b). The initial transient and subsequent sustained signals are, on a different time scale, reminiscent of electrophysiological human and animal response patterns (Pantev et al., 1991; Wang et al., 2005). However, analogies between BOLD signal responses and underlying neural activity should be drawn carefully. Nevertheless, studies which simultaneously recorded intracortical electrophysiological activity and BOLD signal responses have shown the BOLD signal to be correlated with local field potentials (LFPs), i.e. the BOLD contrast mechanism seems to correspond directly with the neural response elicited by a stimulus (Logothetis, 2002). LFPs have been suggested to reflect predominantly synaptic events and typically show a transient increase in power after stimulus onset which is then followed by a lower sustained response during the rest of the stimulus presentation (Logothetis, 2002). Even though the precise mechanisms between neural activity and the hemodynamic response need further investigation (but see also Shmuel et al., 2006), in terms of linear system dynamics the time course of the hemodynamic response is considered to be roughly a lowpass-filtered expression of the total neural activity (Logothetis et al., 2001).

Recent work has shown that transient and sustained component in auditory signal response are differentially regulated in different levels of the ascending pathway (Harms and Melcher, 2002) and have different roles in representing the external acoustic environment (Wang et al., 2005). In their study, Wang and colleagues drew the conclusion, that when a sound is heard, the transient discharges across a relatively large population of neurons mark the onset of a sound. Later on, the activation becomes more restricted to neurons that are preferentially driven by the sound. From animal neurophysiology we know that neurons in cat auditory cortex have different temporal patterns of response (Recanzone, 2000), and that two classes of synapses in layer $2 / 3$ neuron exist in rat auditory cortex, whose response characteristic parallels the transient versus sustained response with which thalamic inputs to the auditory cortex respond to acoustic stimuli (Atzori et al., 2001; Zatorre, 2003). The two classes of synaptic connection are reminiscent of the magnocellular and parvocellular pathways in the visual system for processing different kinds of visual stimuli and it remains the question, whether these parallel channels indeed also show different transmission speeds, as the magno- and parvocellular pathways in the visual system do.

Second, the dissociated lateralization pattern of transient and sustained BOLD signal constituents gives further insight into the computational properties and the biological significance of the two independent response constituents. We suggest that the transient response component is not as much driven by the EI cells as the sustained response component is (see below), and therefore does not primarily reflect ILD information processing. The fact that, independent of its spatial source, the onset of an auditory stimulus is processed by both auditory cortices, even though right and left auditory cortices are known to exhibit some specialization and preference for different stimulus characteristics (Zatorre and Belin, 2001), may signify the importance of an unspecific, but rapid kick off of 'sound detection' processes. This appears to be important for general arousal, orienting response and attention allocation. Furthermore 'auditory edges' represented by transient cortical responses at the onset of new auditory stimuli are essential for the segregation of different auditory events (Fishbach et al., 2001, 2003). The following, maybe slower but more elaborated and specific 'stimulus analysis', including sound localization, may be processed by more specialized neurons (Wang et al., 2005).

Initial sound detection requires the analysis of auditory input alone from a short sampling window, i.e. the full sound characteristic can not be assessed in this stage. In the frequency domain, such short temporal information is reflected by broad spectral information (Papoulis, 1977). However, stimuli with a broad spectrum such as band-pass noise bursts are known to activate more preferentially neurons in the belt areas on the lateral surface of the superior temporal gyrus (Rauschecker, 1998; Seifritz et al., 2006; Wessinger et al., 2001), which in humans have been shown to be the predominating source of the transient response component (Seifritz et al., 2002). The peripheral belt displays a cytoarchitecture, called parakoniocortexis, which is less typical than the core and which can be divided into several subfields (Kaas and Hackett, 1998; Rauschecker et al., 1995). This predominance of transient response constituents in the belt regions has been replicated here. A magnetoencephalographic study by Brancuncci and colleagues demonstrated that ipsilateral inhibition in human auditory cortex is present only when the fundamental frequencies of contra- and ipsilateral sound were similar (Brancucci et al., 2004). Considering the fact that the transient response component reflects activity of neurons being more sensitive to broad- than narrow-band stimuli with a well defined center frequency, this is a further explanation for the absence of ipsilateral inhibition and therefore lateralization of the transient response component. 
In conclusion our data suggest that the dissociated lateralization of transient and sustained response components represent an initial bilateral sound-detection process preceding a more specialized sound analysis - including sound localization - process.

\section{Acknowledgment}

Supported by SNF grant \# PP00B-103012.

\section{References}

Atzori, M., Lei, S., Evans, D.I., Kanold, P.O., Phillips Tansey, E., McIntyre, O., McBain, C.J., 2001. Differential synaptic processing separates stationary from transient inputs to the auditory cortex. Nat. Neurosci. 4 , 1230-1237.

Belin, P., Zatorre, R.J., Hoge, R., Evans, A.C., Pike, B., 1999. Event related fMRI of the auditory cortex. NeuroImage 10, 417-429.

Boynton, G.M., Engel, S.A., Glover, G.H., Heeger, D.J., 1996. Linear systems analysis of functional magnetic resonance imaging in human V1. J. Neurosci. 16, 4207-4221.

Brancucci, A., Babiloni, C., Babiloni, F., Galderisi, S., Mucci, A., Tecchio, F., Zappasodi, F., Pizzella, V., Romani, G.L., Rossini, P.M., 2004 Inhibition of auditory cortical responses to ipsilateral stimuli during dichotic listening: evidence from magnetoencephalography. Eur. J. Neurosci. 19, 2329-2336.

Brodal, A., 1981. The Auditory System, 3rd ed. Oxford Univ. Press, New York.

Caird, D., Klinke, R., 1983. Processing of binaural stimuli by cat superior olivary complex neurons. Exp. Brain Res. 52, 385-399.

Clarey, J., Barone, P., Imig, T.J., 1992. Physiology of Thalamus and Cortex. Springer, New York.

Devlin, J.T., Raley, J., Tunbridge, E., Lanary, K., Floyer-Lea, A., Narain, C., Cohen, I., Behrens, T., Jezzard, P., Matthews, P.M., Moore, D.R., 2003. Functional asymmetry for auditory processing in human primary auditory cortex. J. Neurosci. 23, 11516-11522.

Fishbach, A., Nelken, I., Yeshurun, Y., 2001. Auditory edge detection: a neural model for physiological and psychoacoustical responses to amplitude transients. J. Neurophysiol. 85, 2303-2323.

Fishbach, A., Yeshurun, Y., Nelken, I., 2003. Neural model for physiological responses to frequency and amplitude transitions uncovers topographical order in the auditory cortex. J. Neurophysiol. 90, 3663-3678.

Fox, M.D., Snyder, A.Z., Barch, D.M., Gusnard, D.A., Raichle, M.E., 2005a. Transient BOLD responses at block transitions. NeuroImage 28, 956-966.

Fox, M.D., Snyder, A.Z., McAvoy, M.P., Barch, D.M., Raichle, M.E., 2005b. The BOLD onset transient: identification of novel functional differences in schizophrenia. NeuroImage 25, 771-782.

Friston, K.J., Holmes, A.P., Poline, J.B., Grasby, P.J., Williams, S.C., Frackowiak, R.S., Turner, R., 1995. Analysis of fMRI time series revisited. NeuroImage 2, 45-53.

Harms, M.P., Melcher, J.R., 2002. Sound repetition rate in the human auditory pathway: representations in the waveshape and amplitude of fMRI activation. J. Neurophysiol. 88, 1433-1450.

Jancke, L., Buchanan, T., Lutz, K., Specht, K., Mirzazade, S., Shah, N.J., 1999. The time course of the BOLD response in the human auditory cortex to acoustic stimuli of different duration. Brain Res. Cogn. Brain Res. 8, 117-124.

Jancke, L., Wustenberg, T., Schulze, K., Heinze, H.J., 2002. Asymmetric hemodynamic responses of the human auditory cortex to monaural and binaural stimulation. Hear Res. 170, 166-178.

Joris, P.X., Yin, T.C., 1995. Envelope coding in the lateral superior olive. I. Sensitivity to interaural time differences. J. Neurophysiol. 73, $1043-1062$.

Kaas, J.H., Hackett, T.A., 1998. Subdivisions of auditory cortex and levels of processing in primates. Audiol. Neuro-Otol. 3, 73-85.
Krumbholz, K., Schonwiesner, M., Rubsamen, R., Zilles, K., Fink, G.R., von Cramon, D.Y., 2005. Hierarchical processing of sound location and motion in the human brainstem and planum temporale. Eur. J. Neurosci. $21,230-238$.

Lehmann, C., Herdener, M., Esposito, F., Hubl, D., di Salle, F., Scheffler, K., Bach, D.R., Federspiel, A., Kretz, R., Dierks, T., Seifritz, E., 2006. Differential patterns of multisensory interactions in core and belt areas of human auditory cortex. NeuroImage 31, 294-300.

Logothetis, N.K., 2002. The neural basis of the blood-oxygen-leveldependent functional magnetic resonance imaging signal. Philos. Trans. R. Soc. London, Ser. B Biol. Sci. 357, 1003-1037.

Logothetis, N.K., Pauls, J., Augath, M., Trinath, T., Oeltermann, A., 2001. Neurophysiological investigation of the basis of the fMRI signal. Nature 412, 150-157.

Moore, B., 1994. Hearing (Handbook of Perception and Cognition). Academic Press, New York.

Pantev, C., Makeig, S., Hoke, M., Galambos, R., Hampson, S., Gallen, C., 1991. Human auditory evoked gamma-band magnetic fields. Proc. Natl. Acad. Sci. U. S. A. 88, 8996-9000.

Pantev, C., Ross, B., Berg, P., Elbert, T., Rockstroh, B., 1998. Study of the human auditory cortices using a whole-head magnetometer: left vs. right hemisphere and ipsilateral vs. contralateral stimulation. Audiol. NeuroOtol. 3, 183-190.

Papoulis, A., 1977. Signal Analysis. McGraw-Hill.

Pollak, G.D., Burger, R.M., Park, T.J., Klug, A., Bauer, E.E., 2002. Roles of inhibition for transforming binaural properties in the brainstem auditory system. Hear. Res. 168, 60-78.

Rauschecker, J.P., 1998. Cortical processing of complex sounds. Curr. Opin. Neurobiol. 8, 516-521.

Rauschecker, J.P., Tian, B., Hauser, M., 1995. Processing of complex sounds in the macaque nonprimary auditory cortex. Science 268, 111-114.

Recanzone, G.H., 2000. Response profiles of auditory cortical neurons to tones and noise in behaving macaque monkeys. Hear. Res. 150, $104-118$.

Scheffler, K., Bilecen, D., Schmid, N., Tschopp, K., Seelig, J., 1998. Auditory cortical responses in hearing subjects and unilateral deaf patients as detected by functional magnetic resonance imaging. Cereb. Cortex 8, 156-163.

Schneider, P., Scherg, M., Dosch, H.G., Specht, H.J., Gutschalk, A., Rupp, A., 2002. Morphology of Heschl's gyrus reflects enhanced activation in the auditory cortex of musicians. Nat. Neurosci. 5, 688-694.

Seifritz, E., Esposito, F., Hennel, F., Mustovic, H., Neuhoff, J.G., Bilecen, D., Tedeschi, G., Scheffler, K., Di Salle, F., 2002. Spatiotemporal pattern of neural processing in the human auditory cortex. Science 297, $1706-1708$

Seifritz, E., Di Salle, F., Esposito, F., Herdener, M., Neuhoff, J.G., Scheffler, K., 2006. Enhancing BOLD response in the auditory system by neurophysiologically tuned fMRI sequence. NeuroImage 29 , 1013-1022.

Shmuel, A., Augath, M., Oeltermann, A., Logothetis, N.K., 2006. Negative functional MRI response correlates with decreases in neuronal activity in monkey visual area V1. Nat. Neurosci. 9, 569-577.

Talairach, J., Tournoux, P., 1988. Co-Planar Stereotaxic Atlas of the Human Brain: 3-Dimensional Proportional System: An Approach to Cerebral Imaging. Thieme, Stuttgart.

Tardif, E., Murray, M.M., Meylan, R., Spierer, L., Clarke, S., 2006. The spatio-temporal brain dynamics of processing and integrating sound localization cues in humans. Brain Res. 1092, 161-176.

Wang, X., Lu, T., Snider, R.K., Liang, L., 2005. Sustained firing in auditory cortex evoked by preferred stimuli. Nature 435, 341-346.

Wessinger, C.M., VanMeter, J., Tian, B., Van Lare, J., Pekar, J., Rauschecker, J.P., 2001. Hierarchical organization of the human auditory cortex revealed by functional magnetic resonance imaging. J. Cogn. Neurosci. 13, 1-7.

Woldorff, M.G., Tempelmann, C., Fell, J., Tegeler, C., Gaschler-Markefski, B., Hinrichs, H., Heinz, H.J., Scheich, H., 1999. Lateralized auditory 
spatial perception and the contralaterality of cortical processing as studied with functional magnetic resonance imaging and magnetoencephalography. Hum. Brain Mapp. 7, 49-66.

Yousry, T., Fesl, G., Büttner, A., Noachtar, S., Schmid, U.D., 1997. Heschl's Gyrus. Anatomic description and methods of identification on magnetic resonance imaging. Int. J. Neuroradiol. 5, 2-12.
Zador, A.M., 2001. Synaptic connectivity and computation. Nat. Neurosci. 4, 1157-1158.

Zatorre, R.J., 2003. Sound analysis in auditory cortex. Trends Neurosci. 26, 229-230.

Zatorre, R.J., Belin, P., 2001. Spectral and temporal processing in human auditory cortex. Cereb. Cortex 11, 946-953. 